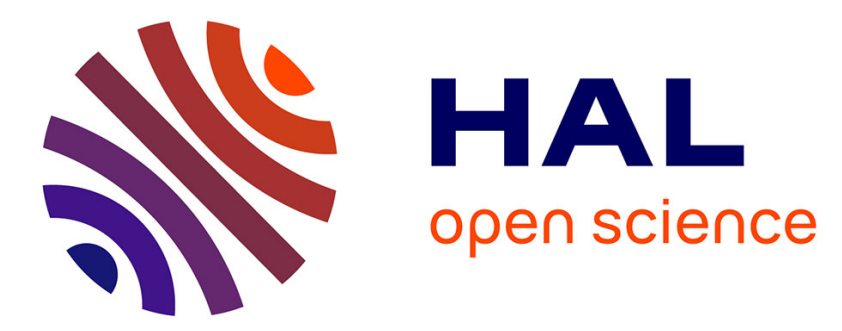

\title{
Combined frequency-tagging EEG and eye tracking reveal reduced social bias in boys with autism spectrum disorder
}

Sofie Vettori, Milena Dzhelyova, Stephanie van Der Donck, Corentin Jacques, Tim van Wesemael, Jean Steyaert, Bruno Rossion, Bart Boets

\section{To cite this version:}

Sofie Vettori, Milena Dzhelyova, Stephanie van Der Donck, Corentin Jacques, Tim van Wesemael, et al.. Combined frequency-tagging EEG and eye tracking reveal reduced social bias in boys with autism spectrum disorder. Cortex, 2020, 125, pp.135-148. 10.1016/j.cortex.2019.12.013 . hal-02930924

\section{HAL Id: hal-02930924 \\ https://hal.science/hal-02930924}

Submitted on 27 Nov 2020

HAL is a multi-disciplinary open access archive for the deposit and dissemination of scientific research documents, whether they are published or not. The documents may come from teaching and research institutions in France or abroad, or from public or private research centers.
L'archive ouverte pluridisciplinaire HAL, est destinée au dépôt et à la diffusion de documents scientifiques de niveau recherche, publiés ou non, émanant des établissements d'enseignement et de recherche français ou étrangers, des laboratoires publics ou privés. 


\title{
Special Issue "Understanding Others": Research Report Combined frequency-tagging EEG and eye tracking reveal reduced social bias in boys with autism spectrum disorder
}

\author{
Sofie Vettori ${ }^{a, b, *}$, Milena Dzhelyova ${ }^{b, c}$, Stephanie Van der Donck ${ }^{a, b}$, \\ Corentin Jacques $^{a, c}$, Tim Van Wesemael ${ }^{d}$, Jean Steyaert ${ }^{a, b}$, \\ Bruno Rossion ${ }^{c, e, f}$ and Bart Boets ${ }^{a, b}$ \\ ${ }^{a}$ Center for Developmental Psychiatry, Department of Neurosciences, KU Leuven, Belgium \\ ${ }^{\mathrm{b}}$ Leuven Autism Research (LAuRes), KU Leuven, Leuven, Belgium \\ c Institute of Research in Psychological Science, Institute of Neuroscience, University of Louvain, Belgium \\ d Department of Electrical Engineering (ESAT), Stadius Center for Dynamical Systems, Signal Processing and Data \\ Analytics, Leuven, Belgium \\ e Université de Lorraine, CNRS, CRAN - UMR 7039, F-54000, Nancy, France \\ ${ }^{\mathrm{f}}$ Université de Lorraine, CHRU-Nancy, Service de Neurologie, F-54000, France
}

\section{A R T I C L E I N F O}

\section{Article history:}

Received 31 May 2019

Reviewed 04 September 2019

Revised 19 September 2019

Accepted 16 December 2019

Action editor Sarah Weigelt

Published online 28 December 2019

Keywords:

Face processing

Frequency-tagging

EEG

Autism spectrum disorder

Eye tracking

\begin{abstract}
A B S T R A C T
Developmental accounts of autism spectrum disorder (ASD) state that infants and children with ASD are spontaneously less attracted by and less proficient in processing social stimuli such as faces. This is hypothesized to partly underlie social communication difficulties in ASD. While in some studies a reduced preference for social stimuli has been shown in individuals with ASD, effect sizes are moderate and vary across studies, stimuli, and designs. Eye tracking, often the methodology of choice to study social preference, conveys information about overt orienting processes but conceals covert attention, possibly resulting in an underestimation of the effects. In this study, we recorded eye tracking and electroencephalography (EEG) during fast periodic visual stimulation to address this issue. We tested 21 boys with ASD (8-12 years old) and 21 typically developing (TD) control boys, matched for age and IQ. Streams of variable images of faces were presented at $6 \mathrm{~Hz}$ alongside images of houses presented at $7.5 \mathrm{~Hz}$ or vice versa, while children were engaged in an orthogonal task. While frequency-tagged neural responses were larger in response to faces than simultaneously presented houses in both groups, this effect was much larger in TD boys than in boys with ASD. This group difference in saliency of social versus non-social processing is significant after 5 sec of stimulus presentation and holds throughout the entire trial. Although there was no interaction between group and stimulus category for simultaneously recorded eye-tracking data, eye tracking and EEG measures were strongly correlated. We conclude that frequency-tagging EEG, allowing monitoring of both overt and covert processes, provides a fast, objective and reliable measure of decreased preference for social information in ASD.
\end{abstract}

(c) 2019 Published by Elsevier Ltd.

\footnotetext{
* Corresponding author. Center for Developmental Psychiatry, Department of Neurosciences, KU Leuven, Belgium.

E-mail address: sofie.vettori@kuleuven.be (S. Vettori).
} 


\section{Introduction}

Newborns and three-month-olds preferentially track moving faces, in contrast to other patterns of comparable complexity, including upside-down faces (Goren, Sarty, \& Wu, 1975; Johnson, Dziurawiec, Ellis, \& Morton, 1991). Over development, this preference for social information sustains. Studies have shown that school-aged children are faster to orient towards faces and attend longer to it (Fischer et al., 2016; Freeth, Ropar, Mitchell, Chapman, \& Loher, 2011; Shaffer et al., 2017; Wilson, Brock, \& Palermo, 2010). However, some studies suggest that this attentional bias decreases over time (Nishizato, Fujisawa, Kosaka, \& Tomoda, 2017) or is context-dependent (Parish-Morris et al., 2013). Likewise, also in adults, there is evidence that attention is rapidly captured by human faces (Crouzet, Kirchner, \& Thorpe, 2010; Fletcher-Watson, Findlay, Leekam, \& Benson, 2008; Hershler \& Hochstein, 2005; Shah, Gaule, Bird, \& Cook, 2013). For example, masked faces are detected more quickly and accurately than masked objects (Purcell \& Stewart, 1988), observers saccade rapidly and automatically towards faces when they appear next to other objects such as vehicles (Crouzet et al., 2010), and changes to faces are better detected than changes to non-face objects (Kikuchi, Senju, Tojo, Osanai, \& Hasegawa, 2009; Rosa Salva, Farroni, Regolin, Vallortigara, \& Johnson, 2011).

Individuals with autism spectrum disorder (ASD) are characterized by impairments in social communication and interaction, and the presence of restricted and repetitive patterns of interests and behavior. They often struggle with social interactions in daily life (American Psychiatric Association, 2013). The social motivation theory of autism (Chevallier, Kohls, Troiani, Brodkin, \& Schultz, 2012) proposes a developmental cascade in which early-onset impairments in social attention initiate developmental processes that may ultimately deprive children of adequate social learning experiences. As a result, an imbalance in attending to social and non-social stimuli might develop, further disrupting social skills and social cognition development, and ultimately social functioning and interaction. Alternatively, according to the 'eye-contact avoidance' hypothesis, individuals with ASD may actively avoid social interactions and eye contact because it is experienced as a highly unpleasant, distressing or even threatening stimulus (Tanaka \& Sung, 2016).

Empirical evidence from eye tracking studies confirms the presence of an atypical imbalance in the attention for social versus non-social stimuli in ASD. Recently, a large-cohort study (Pierce et al., 2016) concluded that enhanced preference for visual stimuli displaying geometric repetition as compared to social stimuli (e.g., videos of playing children) may be an early developmental biomarker of an ASD subtype with more severe symptoms. In a meta-analysis, Frazier and colleagues (Frazier et al., 2017) analyzed and integrated results of 122 independent studies investigating gaze patterns in individuals with ASD as compared to typically developing (TD) individuals. They concluded that individuals with ASD do show a reliable pattern of gaze abnormalities, which suggests a basic problem selecting socially relevant versus irrelevant information. Moreover, gaze abnormalities persist across age and worsen during perception of human interactions. Other meta-analyses report similar evidence for decreased visual attention to social stimuli in individuals with ASD (ChitaTegmark, 2016; Guillon, Hadjikhani, Baduel, \& Rogé, 2014), and demonstrate that an increase in social load, either by including child directed speech or by including several persons interacting with each other, further results in decreased attention to faces in participants with ASD. Generally, eye tracking research thus confirms a reduced looking bias for social stimuli in ASD. However, effect sizes are moderate and vary across studies, stimuli, and designs (Chita-Tegmark, 2016; Guillon et al., 2014).

Yet, a preference for social information comprises more than overt looking behavior. Social attention may also comprise covert orienting, which may not necessarily converge with overt orienting (Petersen \& Posner, 2012; Posner \& Petersen, 1990). Indeed, an individual can covertly orient to a stimulus and process it without exhibiting observable behavior, or -even stronger-an individual may overtly avoid a particular stimulus exactly because internally (covertly) it is processed too strong. For instance, people with spider phobia initially show an early reflexive attentional bias toward threat-related stimuli, followed by avoidance of the threatrelated stimuli (Rinck \& Becker, 2006). In a similar vein, individuals with ASD may covertly avoid social information because it is experienced as too unpleasant or distressing (cf. the eye-contact avoidance hypothesis, Tanaka \& Sung, 2016).

At the neural level, the saliency of this covert orienting and subsequent processing of social information is mostly studied through the N170 (or M170 in MEG), a face-sensitive ERP component peaking at about $170 \mathrm{~ms}$ over occipito-temporal sites following the sudden onset of a face stimulus ((Bentin, Allison, Puce, Perez, \& McCarthy, 1996; Halgren, Raij, Marinkovic, Jousmäki, \& Hari, 2000); for review see; Rossion \& Jacques, 2011). An extensive amount of research has investigated how the N170 may be different in individuals with ASD versus TD controls. A recent meta-analysis pointed to a small but significant delay in N170 latency in response to faces in ASD compared to TD controls (Kang et al., 2018). However, the specificity of the effect is questioned, as it may reflect the generally slower processing of meaningful, even non-social, visual stimuli (Vettori, Jacques, Boets, \& Rossion, 2019).

While eye-tracking measures convey where participants are fixating, stimuli outside the focus of overt attention may still be processed, possibly to a lesser extent. This motivates the combination of simultaneous eye-tracking and EEG recordings to capture both overt and covert processing. However, a particular limitation of a standard ERP approach is the need for slow and sequential stimulus presentation, as the respective responses to each of multiple rapidly simultaneously presented stimuli cannot be disentangled. To address this issue, we used an EEG frequency-tagging approach, where different stimuli are presented simultaneously at different stimulation frequencies, allowing to disentangle the respective neural responses evoked by the different stimuli. The technique is based on the fairly old observation that a visual stimulus presented at a fixed rate, e.g., a light flickering on/off 17 times per second $(17 \mathrm{~Hz})$, generates an electrical brain wave 
exactly at the stimulation frequency (i.e., $17 \mathrm{~Hz}$ in this instance), which can be recorded over the visual cortex (Adrian \& Matthews, 1934). By transforming the data in the frequency domain through Fourier analysis (Regan, 1966), a highly sensitive (i.e., high signal-to-noise ratio, SNR) (Regan, 1989) and objective (i.e., at a pre-determined frequency) quantifiable marker of automatic visual processes without explicit task demands is provided. Moreover, by assigning different tags (frequencies) to different stimuli in the multiinput stimulation, the respective responses corresponding to each of the stimulation frequencies can be disentangled ("frequency-tagging", Regan and Heron, 1969). Hence, evoked responses from populations of cells that are selective to each of the unique input stimuli can be extracted, even if they are spatially overlapping or embedded within the same stimulus event (Norcia, Appelbaum, Ales, Cottereau, \& Rossion, 2015; Rossion \& Boremanse, 2013).

EEG frequency-tagging is particularly suited to study visual spatial attention, as it provides a high-SNR measure of neural activity that can be unambiguously associated with specific stimuli, even when multiple stimuli are present at the same time. Importantly, it also allows monitoring of responses to stimuli that are outside of the focus of attention, which is difficult to obtain with behavioral methods. Changes in amplitude of the markers represent dynamic neural changes related to the processing of the driving stimulus. For instance, the amplitude of the neural response is reliably enhanced when a stimulus is attended versus ignored (Andersen, Fuchs, \& Müller, 2011; Morgan, Hansen, \& Hillyard, 1996; Müller et al., 2006; Vialatte, Maurice, Dauwels, \& Cichocki, 2010). This is supported by previous work stating that the effect of spatial attention on the neural response is not inherently all-ornothing. The magnitude of the visuocortical activity measured with frequency-tagging scales with the amount of attention paid to competing items (Toffanin, de Jong, Johnson, \& Martens, 2009), and is modulated by additional cognitive factors such as memory or emotion (Thigpen, Bradley, \& Keil, 2018).

Likewise, the emotional and social content of visual scenes also modulates the amplitude of the neural responses obtained with frequency tagging. With different paradigms, it has been shown that increasing the emotional content of stimuli increases the amplitude of the neural response (Bekhtereva, Pritschmann, Keil, \& Müller, 2018; Keil et al., 2003). The amplitude of neural responses might also be modulated by subject-specific characteristics. For instance, studies using frequency-tagging have shown that children with ASD show reduced neural responses to brief changes in identity or expression ((Van der Donck et al., 2019; Vettori et al., 2019)). On the other hand, multi-input paradigms in adults with high social anxiety reveal an increased competition from task-irrelevant simultaneously presented threatening faces when executing a concurrent change-detecting task (Wieser, McTeague, \& Keil, 2012).

In the present study we apply EEG frequency-tagging in combination with eye tracking to measure both covert and overt orienting and the subsequent processing of social versus non-social stimuli in individuals with ASD and TD controls. We present two streams of widely varying images of faces and houses, tagged at different frequency rates, simultaneously and next to each other. With eye tracking, we measure the fixations within specific areas of interest spanning each stimulus type. With EEG, we measure the amplitude of the response to each of the stimulus types, as tagged by the presentation frequencies. To our knowledge, this is the first study using frequency-tagging of multiple simultaneous visual inputs in children with ASD and TD children.

In general, we expect that conclusions from neural (EEG) and behavioral (eye tracking) measures will largely correspond. However, as an individual can covertly orient to a stimulus without exhibiting observable behavior (Petersen \& Posner, 2012; Posner \& Petersen, 1990) and stimuli outside the focus of attention can be processed (Toffanin et al., 2009), we expect that both measures will also offer unique information.

In TD children, we expect to find a strong social bias, as indicated by higher proportional looking times (eye tracking) and larger amplitudes (EEG) in response to face stimuli. Based on the literature, compared to TD children, we expect that children with ASD will show a reduced social bias, or that the social bias may be absent. Due to the large variety of eye tracking results, we cannot make predictions about the strength of this effect. As this is the first study using multiinput frequency-tagging in ASD, we do not have a specific hypothesis about the magnitude of the neural effects. On the one hand, neural responses to social stimuli may be reduced in ASD, possibly due to a lack of social interest. On the other hand, neural responses to social stimuli may actually be increased in ASD, possibly because they may be experienced as too arousing and intrusive.

\section{Material and methods}

\subsection{Participants}

We recruited 47 boys, aged 8-to-12 years old. To match the groups on verbal and performance IQ (VIQ, PIQ) five participants (two from the TD group, three from the ASD group) were excluded from the reported analyses, resulting in a sample of 21 typically developing (TD) boys (mean age $=11.0$ years $\pm \mathrm{SD}=1.2$ ) and 21 boys with ASD (mean age $=10.9 \pm 1.5$, Table 1 ). However, inclusion of these participants did not change any results of the analyses. The sample in this study is partially overlapping with the one in our previous study (Vettori et al., 2019), with the exception of six boys with ASD and six TD boys. All participants had normal or corrected-to-normal vision, and had a verbal and performance IQ above 80. 39 participants were right-handed. Participants with ASD were recruited through the Autism Expertise Center of the University Hospitals Leuven, Belgium. TD participants were recruited through elementary schools and sports clubs.

Participant exclusion criteria were the presence or suspicion of a psychiatric, neurological, learning or developmental disorder (other than ASD or comorbid ADHD in ASD participants) in the participant or in a first- or second-degree relative. Inclusion criteria for the ASD group were a formal diagnosis of ASD made by a multidisciplinary team in a standardized way according to DSM-IV-TR or DSM-5 criteria (American Psychiatric Association, 
Table 1 - Participant characteristics.

\begin{tabular}{|c|c|c|c|c|}
\hline & ASD (mean \pm SD) & $\mathrm{TD}($ mean $\pm \mathrm{SD})$ & $t(d f)$ & $p$ \\
\hline Verbal IQ & $107 \pm 12$ & $112 \pm 12$ & $t(40)=-1.41$ & .18 \\
\hline Performance IQ & $104 \pm 15$ & $110 \pm 14$ & $t(40)=-1.44$ & .21 \\
\hline Age & $10.8 \pm 1.6$ & $11 \pm 1.2$ & $\mathrm{t}(40)=.80$ & .43 \\
\hline Social Responsiveness Scale (T-score) & $85 \pm 12$ & $42 \pm 6$ & $t(40)=14.57$ & $<.0001$ \\
\hline
\end{tabular}

2013) and a total T-score above 60 on the Social Responsiveness Scale [SRS parent version (Constantino \& Gruber, 2012)]. Seven participants with ASD took medication to reduce symptoms related to ASD and/or ADHD (Rilatine, Concerta, Aripiprazol). The TD sample comprised healthy volunteers, matched on age, verbal and performance IQ. Parents of the TD children also completed the SRS questionnaire to exclude the presence of substantial ASD symptoms. Descriptive statistics for both groups are displayed in Table 1 , showing that they did not differ for age and IQ. Evidently, both groups differed highly significantly on SRS scores.

The Medical Ethical Committee of the university hospital approved the study, and the participants as well as their parents provided informed consent according to the Declaration of Helsinki. All participants received a monetary reward and a small present of their choice. The experiment was embedded in a larger research project consisting of three testing sessions. Intellectual abilities were assessed in a separate session. The current FPVS experiment was included in the third session.

\subsection{IQ measures}

An abbreviated version of the Dutch Wechsler Intelligence Scale for Children, Third Edition [WISC-III-NL; (Kort et al., 2005; Wechsler, 1991)] was administered. Performance IQ was estimated by the subtests Block Design and Picture Completion, verbal IQ by the subtests Vocabulary and Similarities (Sattler, 2001).

\subsection{FPVS EEG and eye tracking experiment}

\subsubsection{Stimuli}

48 color images of faces and 48 images of houses were used, all within their original background, making the images widely variable. Stimuli were selected from (Retter \& Rossion, 2016) and (Jacques, Retter, \& Rossion, 2016). Examples of stimuli are available in Fig. 1. Faces and houses were presented side-byside on the screen, with a broad rectangular outline around them (Fig. 1): one stimulation stream presented faces, and the other houses. All images differed in terms of viewpoint, lighting conditions and background. All stimuli were resized to $250 \times 250$ pixels, had equal pixel luminance and root-meansquare contrast on the whole image. Shown at a distance of $60 \mathrm{~cm}$, rescaled to $50 \%$ and at a resolution of $1920 \times 1200$, the stimuli each subtended approximately $6.5^{\circ}$ of visual angle. The outer border of the stimuli were separated by $1.5 \mathrm{~cm}$, a width corresponding to a visual angle of $1.4^{\circ}$. Both the face and the object images were presented in a random order.

\subsubsection{Procedure}

After electrode-cap placement, participants were seated at a viewing distance of $60 \mathrm{~cm}$ and were instructed to maintain a constant distance. Stimuli were displayed on the screen (24in. LED-backlit LCD monitor) through sinusoidal contrast modulation on a light grey background using Java. We used a screen with a refresh rate of $60 \mathrm{~Hz}$, ensuring that the refresh

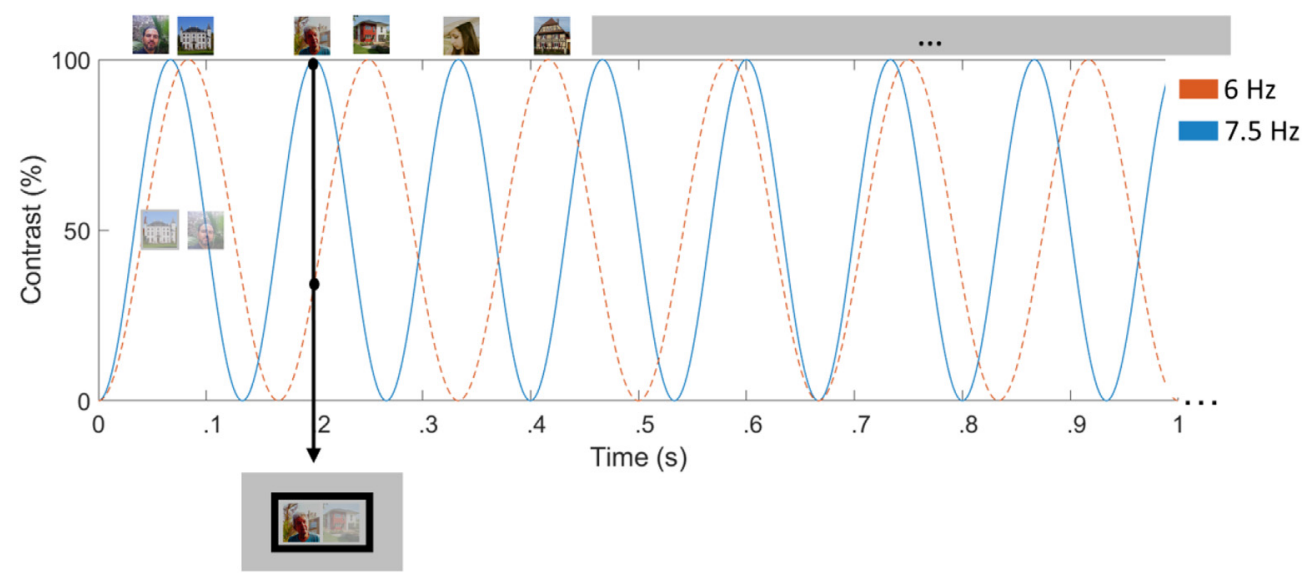

Fig. 1 - Illustration of a sequence. The total experiment consisted of 4 sequences of 30 sec. We counterbalanced frequencies ( 6 and $7.5 \mathrm{~Hz}$ ) and positions of the stimuli (left - right). In the illustrated example, images of houses were presented at $6 \mathrm{~Hz}$, while images of faces were presented at $7.5 \mathrm{~Hz}$. In the other 2 trials, faces were presented at $6 \mathrm{~Hz}$ (right) and houses at $7.5 \mathrm{~Hz}$

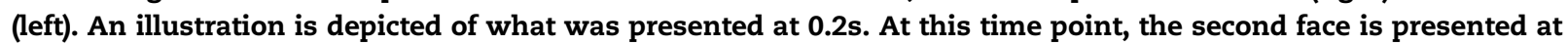
approximately $100 \%$ contrast, while the second house is presented at approximately $30 \%$ contrast. 
rate was an integer multiple of the presentation frequencies. A sequence lasted $34 \mathrm{sec}$, including $30 \mathrm{sec}$ of stimulation at full contrast flanked by 2 sec of fade-in and fade-out, with contrast gradually increasing and decreasing, respectively. Fade-in and fade-out were used to avoid abrupt eye movements and eye blinks due to the sudden appearance or disappearance of flickering stimuli. In total, there were four sequences, hence the total duration of the stimulus presentation was about $2 \mathrm{~min}$.

Fig. 1 illustrates a sequence, comprising of two streams of simultaneously presented series of images. In each sequence, images of one stimulus category were presented at $6 \mathrm{~Hz}$ and images of the other category at $7.5 \mathrm{~Hz}$. One stream of images was shown in the left visual field and the other in the right visual field. All images were drawn randomly from their respective categories, cycling through all available images before any image repetition. The presentation rate $(6 \mathrm{~Hz}$ us $7.5 \mathrm{~Hz}$ ) and the position of the stimuli (left us right) were counterbalanced across both stimulus types (faces vs houses), resulting in 4 sequences presented in a randomized order. The presentation frequencies were selected so that the two stimulation frequencies are close to each other and so that they could not be associated with large differences in absolute EEG response (Boremanse, Norcia, \& Rossion, 2014; Norcia et al., 2015; Regan, 1989).

Participants were instructed to look freely at the images on the screen and to press a key whenever they detected brief (500 msec) changes in the color of the rectangular outline surrounding the images. These color changes occurred randomly, 7 times per sequence. This task was orthogonal to the effect/manipulation of interest and ensured that participants maintained a constant level of attention throughout the entire experiment.

\subsection{EEG recording}

EEG was recorded using a BioSemi Active-Two amplifier system with $64 \mathrm{Ag} / \mathrm{AgCl}$ electrodes. During recording, the system uses two additional electrodes for reference and ground (CMS, common mode sense, and DRL, driven right leg). Horizontal and vertical eye movements were recorded using four electrodes placed at the outer canthi of the eyes and above and below the right orbit. The EEG was sampled at $512 \mathrm{~Hz}$.

\subsection{EEG analysis}

\subsubsection{Preprocessing}

All EEG processing was performed using Letswave 6 (https:// www.letswave.org/) and Matlab 2017 (The Mathworks). The study analyses were not pre-registered prior to the research being conducted, but were in line with previously reported frequency-domain analyses (e.g., Dzhelyova, Jacques, \& Rossion, 2017; Vettori et al., 2019). EEG data was segmented in 37 -second segments ( $2 \mathrm{~s}$ before and $5 \mathrm{~s}$ after each sequence), bandpass filtered $(.1-100 \mathrm{~Hz})$ using a fourth-order Butterworth filter, and downsampled to $256 \mathrm{~Hz}$. Next, noisy electrodes were linearly interpolated from the 3 spatially nearest electrodes (not more than $5 \%$ of the electrodes, -i.e., 3 electrodes, were interpolated). All data segments were re-referenced to a common average reference. We did not perform blink correction for any of the participants, since none of the participants blinked excessively, i.e., more than .36 times/second (2 SD above the mean). Note that FPVS yields responses with a high SNR at specific frequency bins, while blink artefacts are broadband and thus do not generally interfere with the responses at the predefined frequency (Regan, 1989). Hence, blink correction (or removal of trials with many blinks) is not systematically performed in such studies (e.g., Rossion \& Jacques, 2011). The (anonymized) dataset are available on reasonable request.

\subsubsection{Frequency-domain analysis}

Preprocessed segments were further cropped to contain an integer number of $1.5 \mathrm{~Hz}$ cycles (i.e., greatest common divisor of both 6 and $7.5 \mathrm{~Hz}$ ), beginning after fade-in and until $29.36 \mathrm{sec}$ (7516 time bins). The resulting segments were averaged per presentation rate (i.e., combining responses for the same category of images presented either left or right on the screen) in the time domain to preserve the complex phase of the response and reduce EEG activity out-of-phase with the stimulation (i.e., noise). The averaged waveforms were transformed into the frequency domain using a Fast Fourier transform (FFT), and the amplitude spectrum was computed with a high spectral resolution $(.039 \mathrm{~Hz}, 1 / 25.36 \mathrm{sec})$ resulting in a very high signal-to-noise ratio (SNR) (Norcia et al., 2015; Regan, 1989).

Due to neural synchronization of the brain to the presentation rate of the stimuli, the recorded EEG contains a signal at frequencies that are integer multiples (harmonics) of the frequencies at which images are presented $(6 \mathrm{~Hz}$ and $7.5 \mathrm{~Hz}$ ) (Fig. 2). We used two measures to describe the response in relation to the noise level: signal-to-noise ratio (SNR) (Fig. 2) (e.g., Liu-Shuang, Norcia, \& Rossion, 2014) and baselinecorrected amplitudes (Retter \& Rossion, 2016) (Fig. 3). SNR spectra were computed for each electrode by dividing the value at each frequency bin by the average value of the 20 neighboring frequency bins (12 bins on each side, i.e., 24 bins, but excluding the 2 bins directly adjacent and the 2 bins with the most extreme values). We computed baseline-corrected amplitudes in a similar way by subtracting the average amplitude of the 20 surrounding bins. For group visualization of topographical maps (Fig. 3), we computed across-subjects averages of the baseline-corrected amplitudes for each condition and electrode separately.

To define the harmonics that were significantly above noise level, we computed Z-score spectra on group-level data for each stimulation frequency (Dzhelyova et al., 2017; Jacques et al., 2016; Liu-Shuang et al., 2014; Rossion, Torfs, Jacques, \& LiuShuang, 2015). We averaged the FFT amplitude spectra across electrodes in the relevant regions-of-interest (ROIs) based on topographical maps, and transformed these values into Zscores (i.e., the difference between the amplitude at each frequency bin and the mean amplitude of the corresponding 20 surrounding bins, divided by the SD of the amplitudes in these 20 surrounding bins). For $6 \mathrm{~Hz}$, Z-scores were significant (i.e., $Z>2.32$ or $p<.01)$ until the 5 th harmonic $(30 \mathrm{~Hz})$ and for $7.5 \mathrm{~Hz}$, 

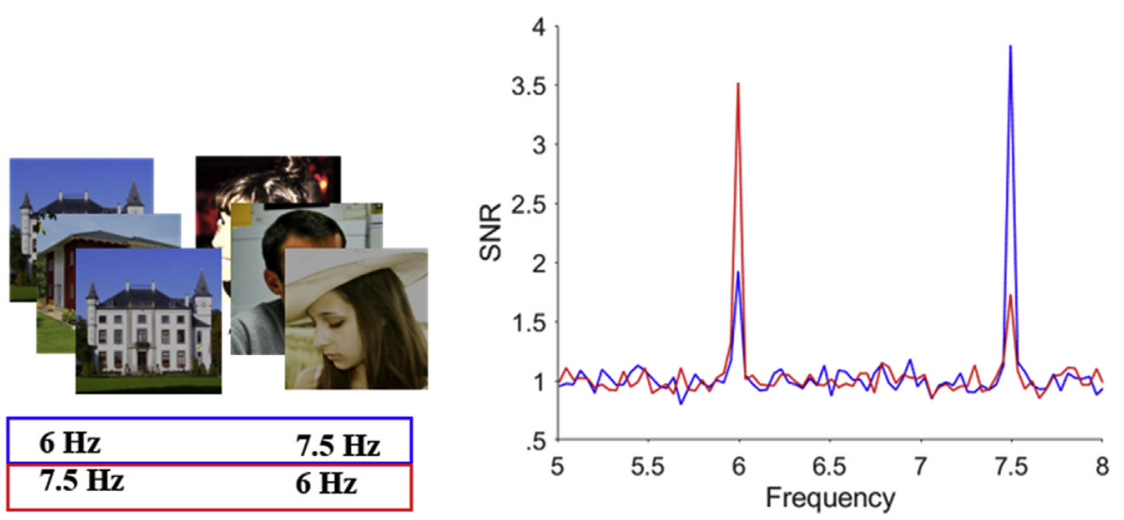

Fig. 2 - SNR spectra for the two presentation frequencies, averaged over all participants. Faces generally elicit a larger frequency-tagged response, in both presentation rate combinations (i.e. red line at $6 \mathrm{~Hz}$ and blue line at $7.5 \mathrm{~Hz}$ ).

Z-scores until the fourth harmonic $(30 \mathrm{~Hz})$ were significant. To include an equal number of harmonics for both stimulation frequencies and to exclude shared harmonics $(30 \mathrm{~Hz})$, we selected the first three harmonics for both frequencies and summed the baseline-corrected amplitudes of those for each frequency and each condition separately.

Hence, we quantified neural responses to faces and houses at $6 \mathrm{~Hz}$ and at $7.5 \mathrm{~Hz}$ by summing the baseline-subtracted responses for 3 harmonics: $6 \mathrm{~Hz}, 12 \mathrm{~Hz}$ and $18 \mathrm{~Hz}$ for the $6 \mathrm{~Hz}$ stimulation frequency; and $7.5 \mathrm{~Hz}, 15 \mathrm{~Hz}$ and $22.5 \mathrm{~Hz}$ for the $7.5 \mathrm{~Hz}$ stimulation frequency. Therefore, we obtained an index of neural saliency per stimulus type (i.e., houses us faces) and per presentation rate.

Based on a priori knowledge, in accordance with previous studies and confirmed by visual inspection of the topographical maps of both groups (Fig. 3), we identified regions of interest (ROI) in which the signal was maximal and averaged the signal at these nearby electrodes. The analysis of the response to both types of stimuli focused on three ROIs: medial occipital (MO: Iz, Oz, POz), left occipitotemporal (LOT: O1, PO7, P7, P9) and right occipito-temporal (ROT: O2, PO8, P8, P10).

\subsubsection{Statistical analysis}

We statistically analyzed the baseline-corrected amplitudes in each ROI for each stimulus type at the grouplevel using general linear mixed models (LMEMs) using the Afex package v0.22-1 (Singmann, Bolker, Westfall, \& Aust, 2018) in R v3.4.3 (R Core Team, 2012). We opted for LMEMs, as they are particularly suited for analyzing data with repeated measurements and are superior compared to, for example, ANOVAs when dealing with unbalanced and missing data (Baayen, Davidson, \& Bates, 2008). In particular, we examined the neural responses (i.e., baseline-subtracted amplitudes) with stimulus type (houses us faces) and ROI (MO, LOT, ROT) as within-subject factors, and group (ASD us TD) as a between-subject factor. We included a random intercept per participant to account for the clustering in the data. We performed a Yeo-Johnson transformation on the data to better meet the assumption of normality of the residuals. Post-hoc Z-tests were performed on the fitted model using the emmeans package (Lenth, Singmann, Love, Buerkner, \& Herve, 2019). Tukey-corrected $p$-values were used to compare means. Additionally, in order to test for power differences at the two stimulation frequencies, we performed a LMEM with Frequency as an additional factor.

In addition, we determined the significance of responses for each individual participant and each stimulus type as follows [e.g., (Dzhelyova et al., 2017; Retter \& Rossion, 2016; Vettori et al., 2019)]: (1) the raw FFT amplitude spectrum was averaged across electrodes per ROI, and (2) cut into segments centered on the harmonics of the target frequency bin (i.e., 6 or $7.5 \mathrm{~Hz}$ ) surrounded by 20 neighboring bins on each side; (3) the amplitude values across the segments of FFT spectra were summed; (4) the summed FFT spectrum was transformed into a $z$-score using the 20 surrounding bins (see above). Responses of a given participant were considered significant if the $z$ score at the target frequency bin exceeded 1.64 (i.e., $p<.05$ one-tailed: signal $>$ noise).

\subsubsection{Time-frequency analysis}

In addition, we were interested in how the neural responses changed over the course of a stimulation sequence. We applied short-term Fourier transformations to the data, with window sizes of $5 \mathrm{sec}$ and with a sliding step of 10 time bins. This computation was applied on pre-processed EEG epochs for each subject and condition (after re-referencing). Next, we averaged the separate epochs across trials for each subject and condition. Finally, we computed the amplitude envelope of the EEG signal across time exactly at the stimulation frequencies (6 and $7.5 \mathrm{~Hz}$ ).

Next, we assessed the evolution of the group difference over time. This was done by cutting the original data in segments of increasing length $(5-30 \mathrm{sec}$ in steps of $5 \mathrm{sec})$ and repeating the frequency analysis that was outlined above. For all segments, we statistically analyzed the baseline-corrected amplitudes in each ROI for each stimulus type at the grouplevel using general linear mixed models, again focusing on the group by stimulus type interaction. 


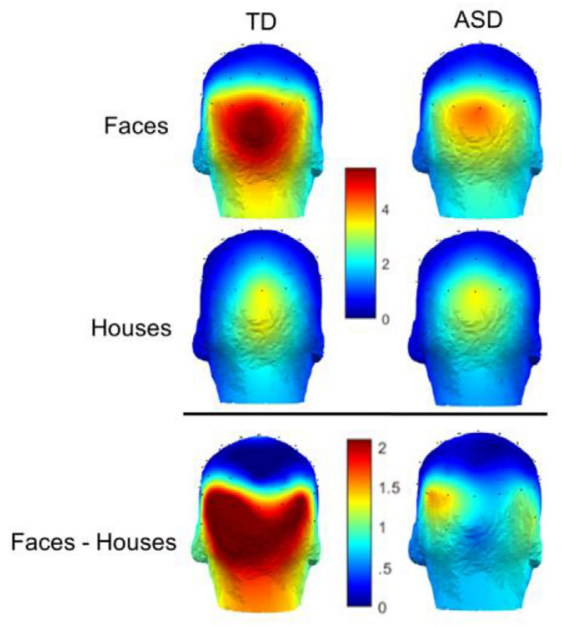

A

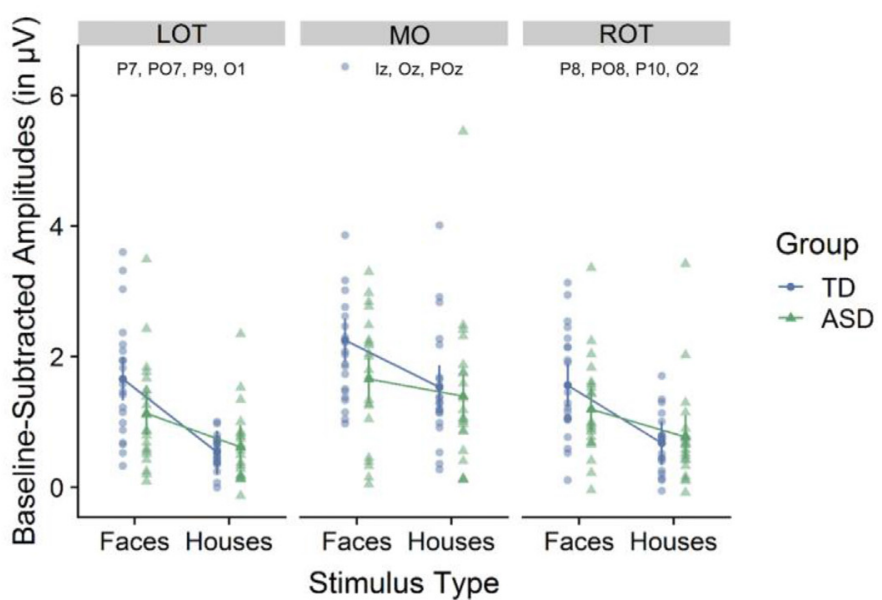

B

Fig. 3 - A. Scalp distribution of the EEG signal during FPVS (baseline subtracted amplitudes in $\mu$ V). Frequency-tagged neural responses to the streams of periodically presented faces and houses are shown for each participant group, as well as the differential response for faces minus houses. While both groups display higher responses to faces than to houses, this difference is larger in the TD group than in the ASD group. B. Averaged baseline-subtracted amplitudes for each stimulus condition (faces us houses) for each group and for each ROI. The individual subject data is displayed in the background. Statistical analysis shows an interaction between group and stimulus type, indicating that the difference between faces and houses is larger in the TD group compared to the ASD group.

\subsection{Eye tracking recording}

Eye tracking data were collected using a Tobii X3-120 screenbased remote eye tracker and Tobii Pro software (Tobii Pro). The sampling rate was $120 \mathrm{~Hz}$. Binocular gaze precision at ideal conditions is estimated at $.24^{\circ}$ of visual angle and binocular gaze accuracy at $.4^{\circ}$. However, for many experiments these ideal conditions are not met (Niehorster, Cornelissen, Holmqvist, Hooge, \& Hessels, 2018). With a remote eye tracker, participants are free to move their head within the "headbox" allowing eye tracking (Niehorster, Cornelissen, Holmqvist, Hooge, \& Hessels, 2018). Due to this freedom of movement, as well as the use of pediatric and/or patient populations, the precision and accuracy of the actual data may differ from those marketed by the manufacturers. In the standard calibration procedure of the Tobii X3-120, participants have to follow a red dot moving across the screen, yielding a merely qualitative index of calibration quality based on visual inspection. In order to obtain a subject-specific quantitative measure of eye tracking data quality, we implemented an additional calibration validation paradigm, preceding the data registration. In this additional calibration procedure, participants had to fixate on the center of nine consecutive fixation crosses appearing on different locations on the screen. Calculation of the angle between the vectors to the displayed fixation cross versus the actual gaze point yields a quantitative index of error angle (mean and variance) and resulting accuracy. These values were used in the analysis to attribute gaze points to particular areas of interest (AOIs).

\subsection{Eye tracking analysis}

2.7.1. Fixation filter, definition of areas of interest (AOI), gaze attribution

Eye tracking data were analyzed by means of a series of custom-built Matlab scripts (The Mathworks). We used the I2MC algorithm [(Hessels, Niehorster, Kemner, \& Hooge, 2017), identification by 2-means clustering] to filter the raw eye tracking data (i.e., deleting random noise, interpolating missing data, identifying fixations).

In the current study, the two AOIs (faces, houses) were defined as the rectangular areas spanning the two zones where the pictures are shown (see Fig. 4 and description of the stimuli above). In addition, we defined the area 'outside AOI' to comprise all the fixation points that were not assigned to either the face or the house AOI.

Fixations were attributed to the AOIs by means of a probability weighting, taking into account the subjectspecific data quality as obtained via the additional calibration validation procedure. For every gaze point, a proportional score between zero and one is attributed to every AOI (i.e., 'faces', 'houses', 'outside AOI'), in such a way that the cumulative sum of these scores equals one. The size of this score indicates the probability that the corresponding AOI effectively contains the recorded gaze point, taking into account the subject-specific data quality. Assignment of proportional scores depends on a two dimensional bell curve 
A
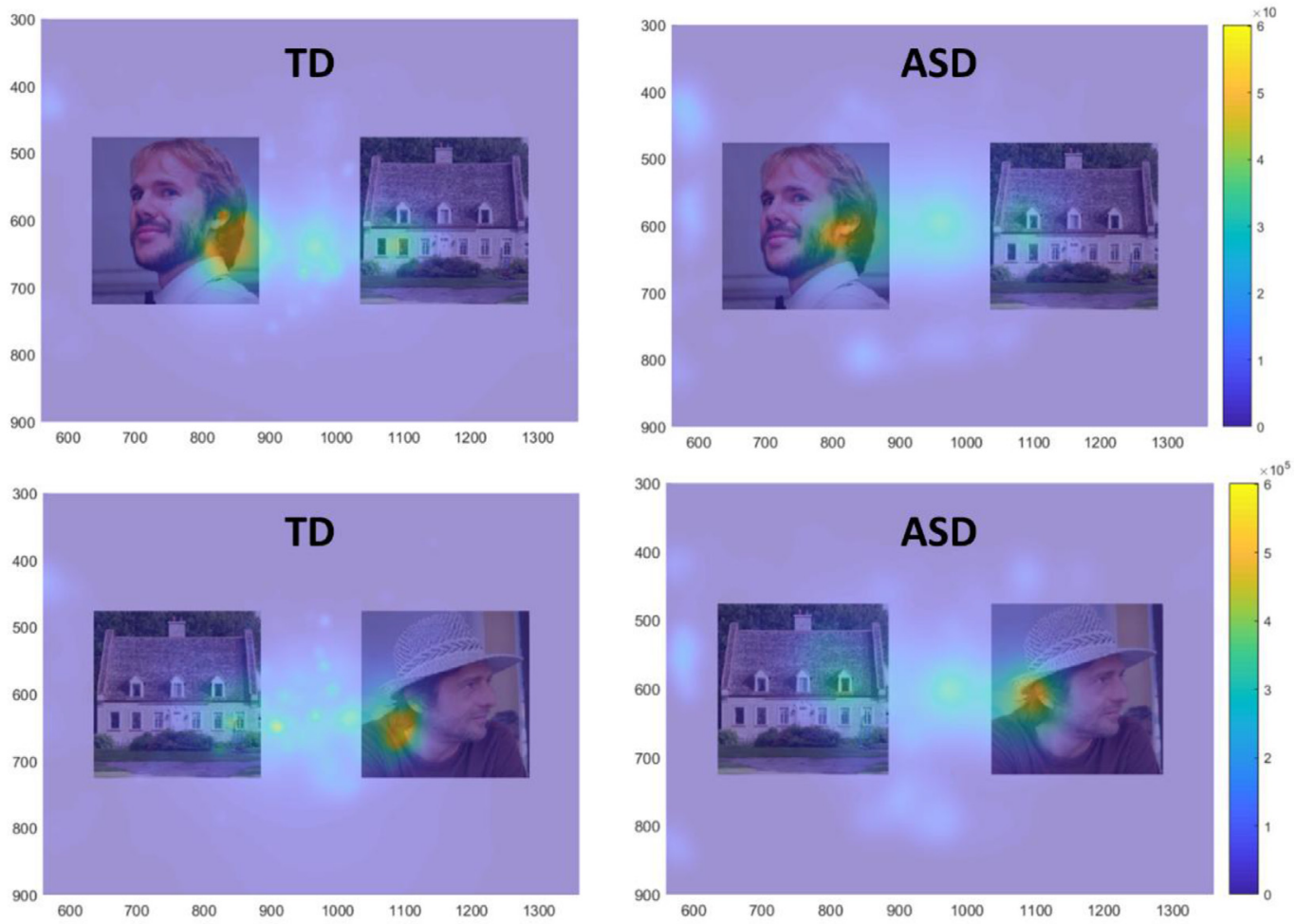

B

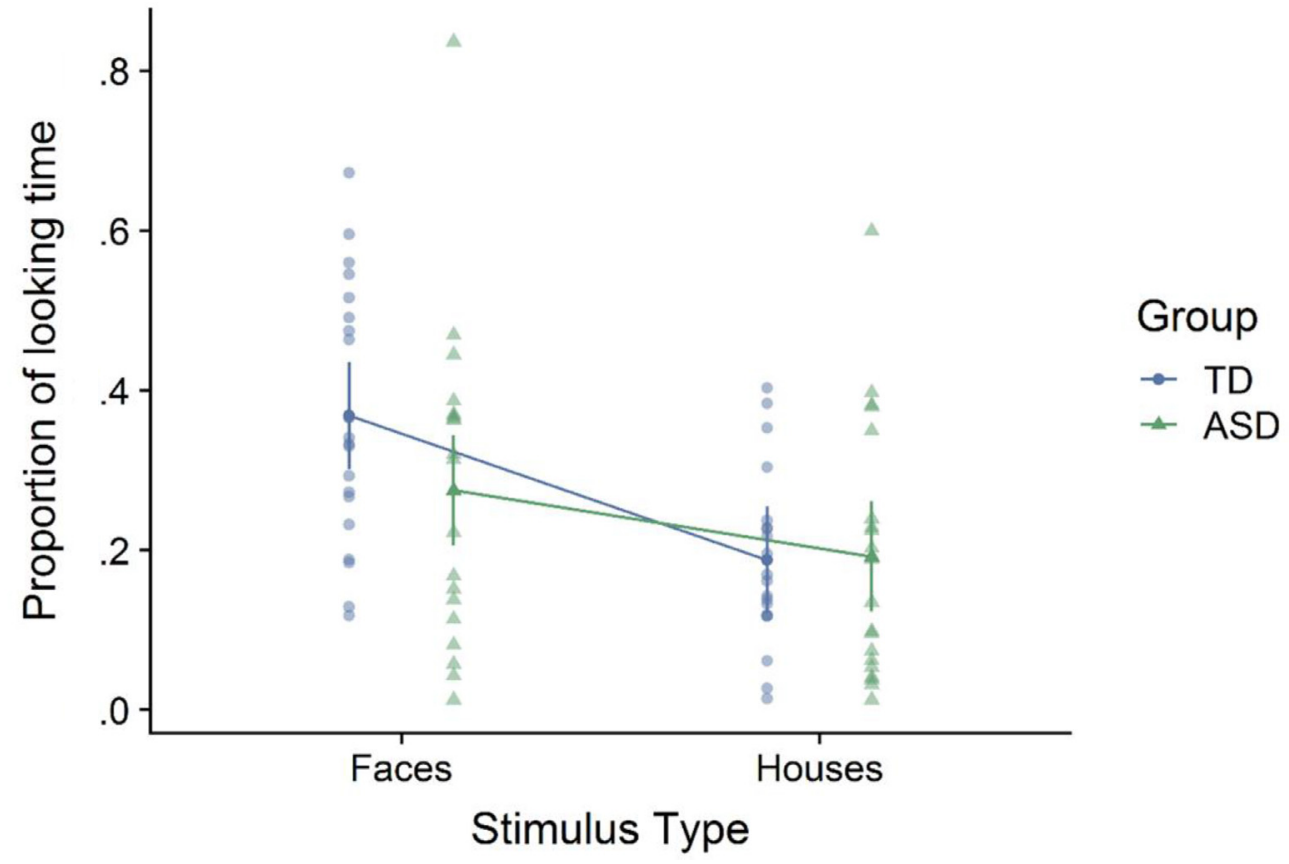

Fig. 4 - A. Heatmap of gaze patterns averaged over all participants of each group. The orange areas indicate longer total looking times. B. The mean and $95 \%$ confidence intervals of the proportion of looking times to faces and houses in each group. 
around the gaze point with a standard deviation equal to the RMS registered during calibration validation. Hence, the calibration validation determines the probability weighting of the AOIs: better data quality results in more concentrated sample points around the gaze point, poorer data quality results in more dispersed sample points. Since the algorithm takes every gaze point into account, as well as the data quality, it proves to be a very reliable method.

For each AOI, the relative duration of all fixation points was averaged over the four trials. Proportional looking times for each of both AOIs were calculated, as well as the proportion of looking time outside the AOIs.

\subsubsection{Statistical analysis}

We analyzed the eye tracking data at the group-level using general linear mixed models using the Afex package (Singmann et al., 2018) in R (R Core Team, 2018). The proportional looking time was examined using stimulus type (faces us houses) as a within-subject factor, and group (ASD vs TD) as a between-subject factor. We included a random intercept per participant. Post-hoc Z-tests were performed on the fitted model using the emmeans package (Lenth et al., 2019). Tukeycorrected $p$-values were used to compare means. For one participant in the ASD group, eye tracking data was not recorded due to technical failure.

\subsubsection{Evolution of the eye tracking signal over time}

Additionally, we were interested in how the proportion of looking times in each AOI changed over time. Therefore, we divided the trial in time segments of $5 \mathrm{sec}$, with sliding steps of $1 \mathrm{sec}$, and computed the probability of looking to each AOI for each of these time segments.

We report how we determined our sample size, all data exclusions, all inclusion/exclusion criteria, whether inclusion/exclusion criteria were established prior to data analysis, all manipulations, and all measures in the study. No part of the study procedures or analysis plans was preregistered in an institutional registry prior to the research being conducted. The conditions of our ethics approval do not permit public archiving of individual anonymized raw data. Readers seeking access to the data should contact the lead author Sofie Vettori and/or the senior author Bart Boets at the Center for Developmental Psychiatry, Department of Neurosciences, University of Leuven. Access will be granted to named individuals in accordance with ethical procedures governing the reuse of sensitive data. Specifically, requestors must complete a formal data sharing agreement to obtain the data. Legal restrictions do not allow to store the face images online, but readers seeking access to the images for research should contact the corresponding author. Access will be granted to named individuals who complete a formal agreement stating that images will only be used for research. The presentation and analysis scripts, and the house images can be found in: https://osf.io/zcu73/.

\section{Results}

\subsection{No group difference in orthogonal task performance}

Both groups performed equally on the behavioral color change detection task, suggesting a similar level of attention throughout the experiments. Both groups showed accuracies between $94(\mathrm{SD}=7.5 \%)$ and $97 \%(\mathrm{SD}=3.2 \%)$ with mean response times between $.51(\mathrm{SD}=.10)$ and $.46(\mathrm{SD}=.04) \mathrm{sec}-$ onds, for ASD and TD respectively. Statistical analyses (twosided t-tests) showed no significant differences between the ASD group and the TD group [accuracy: $\mathrm{t}(25)=-1.83, p=.08$; response times $\mathrm{t}(25)=2, p=.06]$.

\subsection{EEG}

We observed robust frequency-tagged responses, in the three regions of interest (ROI) and for the two stimulus types (see magnitude and scalp distribution in Fig. 3). Analyses at the individual level indicated that, despite the short recording time, all but one participant showed significant responses to houses and to faces in the pre-specified ROIs.

At the group level, statistical analyses showed a highly significant main effect of Stimulus Type $[F(1,452)=47.92$, $p<.0001]$, indicating that on average, the magnitude of the response was higher in response to face stimuli than to house stimuli. There was no main effect of Group $[\mathrm{F}(1,40)=1.34$, $p=.25]$. However, crucially, we observe a highly significant Group by Stimulus Type interaction effect $[F(1,452)=10.63$, $p=.001]$. Post-hoc testing reveals that the response for faces versus houses is much larger in the TD group $(z=9.94$, $\left.p_{\text {corr }}<.0001\right)$ than in the ASD group $\left(z=5.33, p_{\text {corr }}<.0001\right)$. Furthermore, there is no group difference in the response to houses $\left(z=.19, p_{\text {corr }}=.10\right)$ while there is a trend for a larger response to faces in the TD group compared to the ASD group $\left(z=2.35, p_{\text {corr }}=.087\right)$. We also observed a significant main effect of ROI $[F(2,452)=47.92, p<.0001]$, indicating that the magnitude of the response is higher in the MO region compared to left and right OT. However, this effect was modulated by Stimulus Type $[F(2,452)=4.89, p=.008]$, indicating that the differential response for faces versus houses is larger in the lateral occipital areas (LOT: $z=8.30, p_{\text {corr }}<.0001$, ROT: $\left.z=6.50, p_{\text {corr }}<.0001\right)$ than in the medial occipital area $\left(z=3.90, p_{\text {corr }}<.0013\right)$. There were no other significant two- or three-way interactions.

Additionally, we statistically assessed the significance of the effect of Frequency. We observed a significant effect of frequency $[\mathrm{F}(1,440)=12.85 ; p=.0004]$, indicating that responses at $6 \mathrm{~Hz}$ and harmonics are larger than at $7.5 \mathrm{~Hz}$ and harmonics. However, there were no interactions between frequency and any of the other factors [Frequency $\times$ Group: $\mathrm{F}(1,440)=.04, p=.84 ;$ Frequency $\times$ Stimulus Type: $\mathrm{F}(1,440)=.02$, $p=.88$, Frequency $\times$ Stimulus Type $\times$ Group: $\mathrm{F}(1,440)=.61 ;$ $p=.43]$. So in general, for both stimulus types and for both groups, the power at $6 \mathrm{~Hz}$ was larger than at $7.5 \mathrm{~Hz}$, meaning that a particular presentation frequency was not preferred for a certain stimulus type or a particular group. 


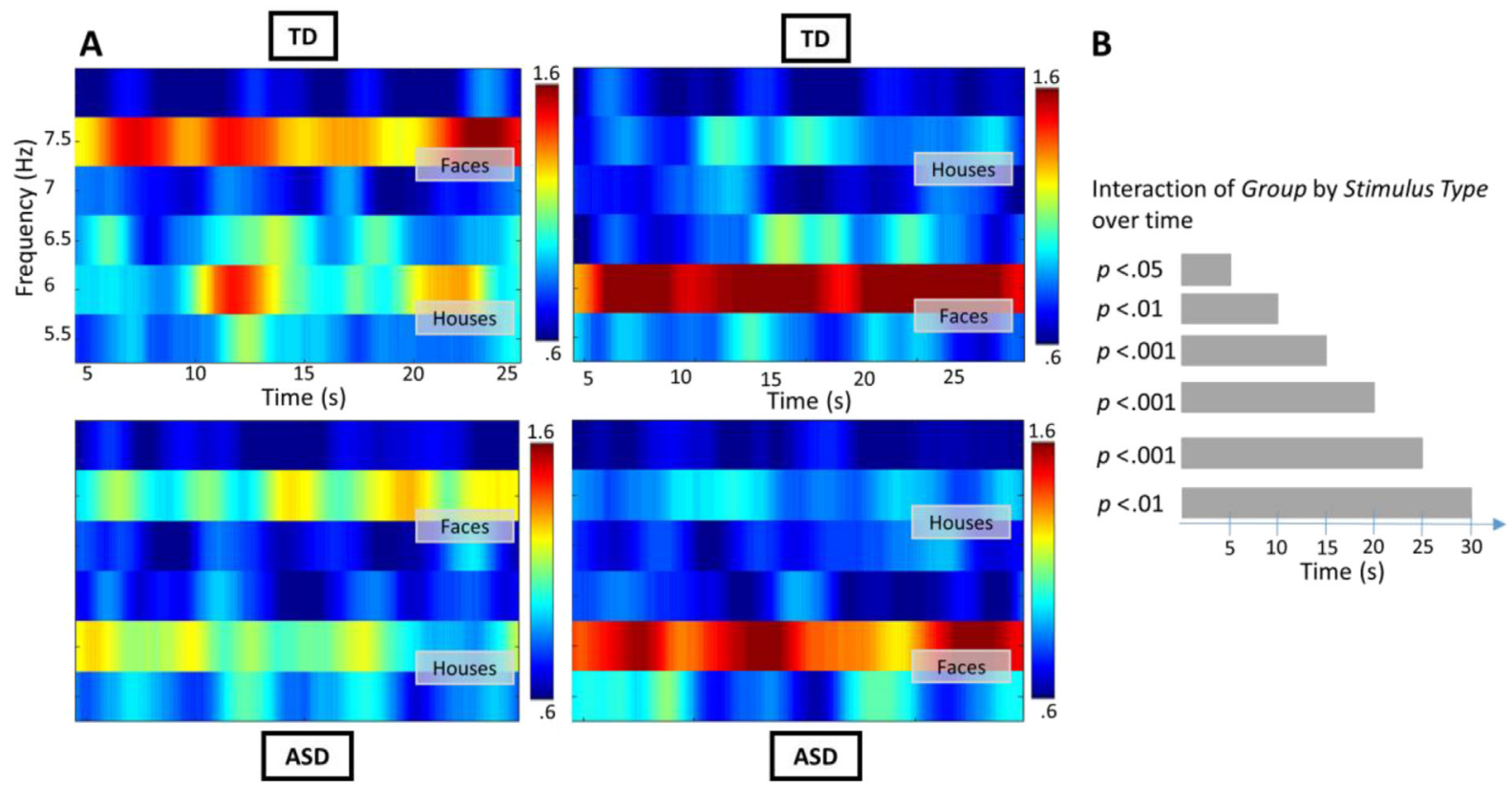

Fig. 5 - A. Time-frequency decomposition of the EEG data based on the short-term Fourier transform averaged across all participants of each group. The response is centered on the frequency of stimulation $(6 \mathrm{~Hz}$ and $7.5 \mathrm{~Hz})$. For both groups the response to faces is larger than the response to houses, but this effect is much more prominent in the TD group compared to the ASD group. The time-frequency decomposition visually shows that the Group by Stimulus type interaction effect is present over the whole trial duration. B. Corresponding $p$-values for the Group by Stimulus type interaction, calculated for incrementing time windows from 5 to $30 \mathrm{sec}$. The interaction effect is immediately significant after 5 sec of stimulus presentation and holds throughout the entire trial duration.

\subsection{Time-frequency analysis of the EEG data}

Fig. 5 shows the results of the time-frequency analysis, averaged across all electrodes of the three ROIs (LOT, MO, ROT). The neural responses do not show adaptation in either of the conditions, remaining stable in amplitude until the end of the sequence. Visually, it can be observed that the increased response to faces compared to houses is most prominent in the TD group, across the whole trial duration (Fig. 5A). To confirm this statistically, we computed linear mixed models testing the Group by Stimulus type interaction effect for each of the time segments with increasing lengths (i.e., 5-10-15-20-25-30 sec). These analyses show that from $5 \mathrm{~s}$ onwards and across each of these time windows, the interaction effect is significant $(p<.05)$, indicating that over the whole course of the trial, the evoked neural response to faces versus houses is higher in TD boys than in boys with ASD (Fig. 5B).

\subsection{Eye tracking}

Analysis of the data quality reveals slight differences between groups. The average error angle was significantly higher in the ASD group $\left(.56^{\circ} \pm .06^{\circ}\right)$ than in the TD group $\left(.40^{\circ} \pm .046^{\circ}\right)$ $[\mathrm{t}(39)=-2.14, p=.038]$. The root-mean-square of this angle however, did not differ between groups [ASD: .68 \pm . 19, TD: $.64 \pm .63 ; \mathrm{t}(39)=-.24, p=.41]$.
In general, participants from both groups gaze a significant amount of time outside both target AOIs. Visual inspection (Fig. 4) of the gaze patterns shows that this is mostly in between the two stimuli. This amount of looking outside the AOIs did not differ between groups $[\mathrm{T}(39)=-1.30, p=.20]$.

Eye tracking data (proportion of looking time to each AOI) are shown in Fig. 4. Statistical analysis shows a main effect of Stimulus Type $[F(1,39)=15.69, p=.0003]$, indicating that both groups look more to faces than to houses. There is no significant main effect of Group $[F(1,39)=2.29, p=.14]$. Unlike in the EEG analysis, and contrary to our hypothesis, the interaction between Group and Stimulus Type was not significant $[F(1,39)=1.86, p=.18]$. However, post-hoc contrasts using a Tukey-Kramer correction revealed that similarly to the EEG data, children in the TD group did look significantly longer to faces compared to houses $\left(Z=3.81, p_{\text {cor }}=.0008\right)$, whereas this stimulus type effect was not significant in the group of children with ASD $\left(Z=1.82, p_{\text {cor }}=.35\right)$.

\subsection{Evolution of the eye tracking signals over time}

To assess the evolution of the proportional looking times in each AOI over time, we divided the trial in time segments of $5 \mathrm{sec}$, with sliding steps of $1 \mathrm{sec}$, and computed the probability of looking to each AOI for each of these time segments. These results are displayed in Fig. 6. We computed LMEMs testing 

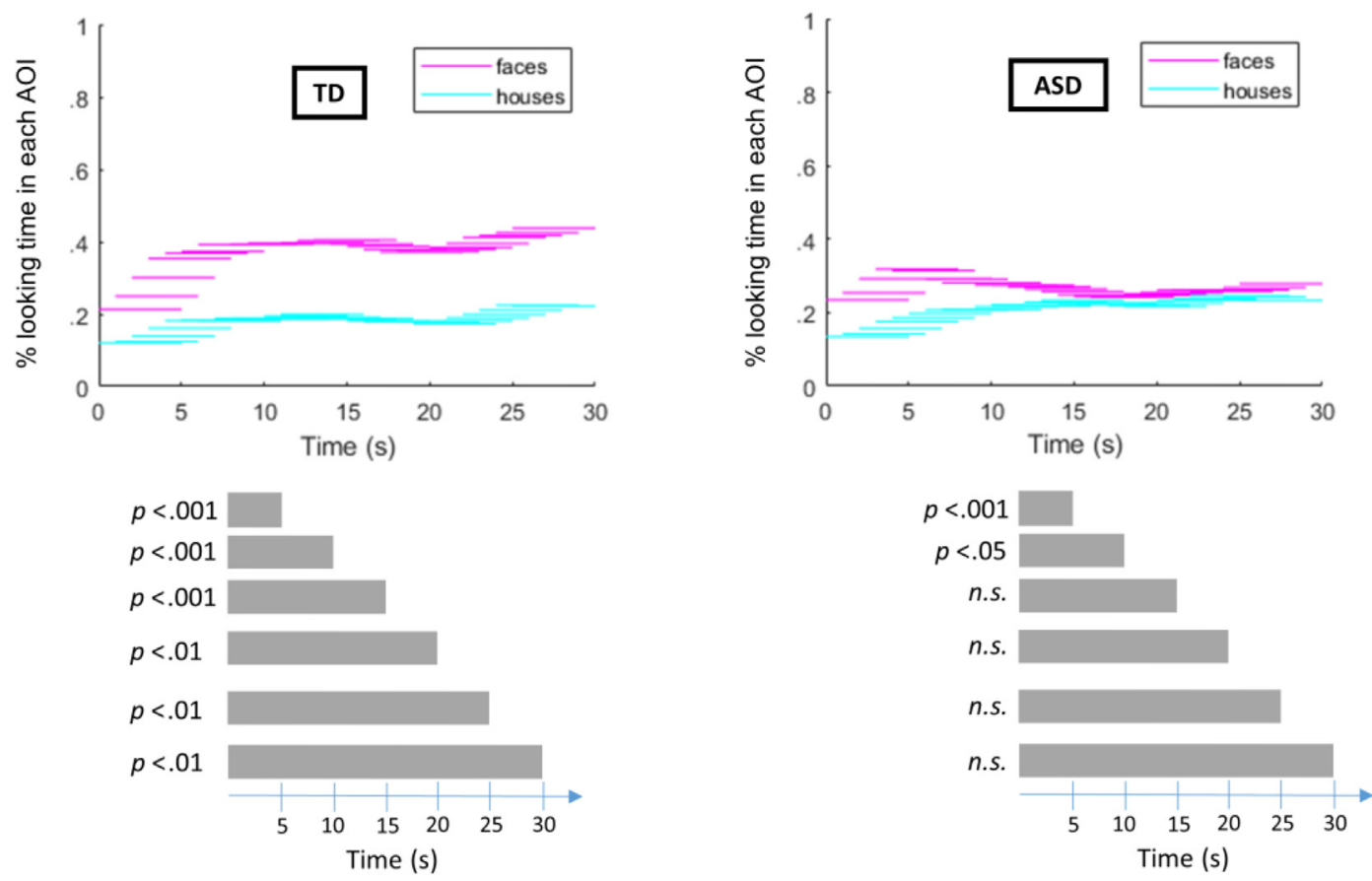

Fig. 6 - Temporal evolution across the $30 \mathrm{sec}$ trial of the proportional looking times to faces and to houses, averaged across sliding $5 \mathrm{~s}$ time windows for each of the participant groups. In each of the time segments, the proportional looking time to faces versus houses is significantly larger in the TD group. In the ASD group, proportional looking times to faces is larger than to houses for time segments of 5 and $10 \mathrm{sec}$. From $15 \mathrm{sec}$ onwards, proportional looking times to houses and faces are equal across the entire trial in ASD.

the Group by Stimulus type interaction effect for each of the time segments with increasing lengths (i.e., 5-10-15-20-25$30 \mathrm{sec}$ ). Analogous to the analysis of the full trial, the interaction between Group and Stimulus Type was not significant in any of the time segments. However, post-hoc contrasts using a Tukey-Kramer correction revealed that in each of the time segments, the proportional looking time to faces versus houses is significantly larger in the TD group. In the ASD group, proportional looking times to faces is larger than to houses for time segments of 5 and $10 \mathrm{sec}$. From $15 \mathrm{sec}$ onwards, proportional looking times to houses and faces are equal across the entire trial in ASD.

\subsection{Correlations between EEG and eye tracking}

In general, over both groups and over the full trial length, individual differences in social bias (i.e., the response to faces minus the response to houses) were highly related when measured by EEG and by eye tracking $\left(r_{\mathrm{P}}=.75, p<.00001\right)$. Both in the ASD group $\left(r_{\mathrm{P}}=.59, p<.001\right)$ and in the TD group $\left(r_{\mathrm{P}}=.74, p<.0001\right)$, this association was highly significant. There was no evidence that the associations were significantly different between groups $(\mathrm{Z}=.82, p=.41)$.

\section{Discussion}

A prominent hypothesis in ASD is that a reduced preference for social information in early life might affect social functioning later on (Chevallier et al., 2012). Social preference in ASD has typically been studied with eye tracking paradigms, allowing the measurement of fixation. This study was motivated by the fact that this approach precludes measuring processing of stimuli outside the focus of overt attention. Thus, in addition to studying overt processes with eye tracking, we used frequency-tagging EEG to study covert processes in TD and ASD boys. Importantly, this approach allows monitoring of responses to stimuli that are outside the attentional locus. Changes in amplitude of the markers represent dynamic neural changes related to the processing of the driving stimulus, more specifically, frequency-tagged streams of variable face and house stimuli at 6 and $7.5 \mathrm{~Hz}$ (and vice-versa).

Here, we observe that school-aged boys with ASD do prefer social (i.e., faces) over non-social (houses) information, but this social preference is much less pronounced as compared to the TD group. Pertaining to the EEG data, both TD and ASD boys show higher neural responses to faces than to houses, but the significant interaction indicates that this stimulus type effect is much smaller in boys with ASD. Although there was no such significant interaction between groups and stimulus category in eye tracking, looking patterns during eye tracking tend to indicate that the preferential looking to faces is less present in boys with ASD. Moreover, these measures are strongly correlated, indicating that reduced looking times co-occur with reduced neural responses, thereby suggesting reduced overt and covert social processing and preferencing in ASD. 
The relatively reduced interest in social processing in ASD that we observed might be related to a reduced sensitivity to the reward value of social stimuli, in line with the social motivation hypothesis (Chevallier et al., 2012). Evidence suggests that social stimuli are less rewarding for individuals with ASD, as seen in diminished neural responses in the neural circuitry supporting reward learning (Zeeland, Dapretto, Ghahremani, Poldrack, \& Bookheimer, 2010). A recent meta-analysis points out that individuals with ASD show a neurobiological difference in response to rewarding social input, which may in turn lead to diminished social motivation. The aberrant processing of rewards extends to non-social stimuli and thereby might underlie their increased motivation for restricted non-social interests (Clements et al., 2018).

Reduced interest in social processing in ASD might result in less frequent engagement with faces. As a result, developing neural systems devoted to face processing would lack experience-expectant visual input, which ultimately may prove necessary for establishing the neural architecture for expert face processing competency (Chevallier et al., 2012). If it is indeed the case that differential experience with faces over the course of development influences the neurological wiring involved in face perception and processing, implementing behavioral interventions that encourage individuals with autism to engage with and process faces may stimulate and benefit affected brain regions (Dawson et al., 2002). This might affect social functioning later on.

Most studies investigating social preference use eye tracking. In this study, we combined frequency-tagging EEG and eye tracking and our results demonstrate convergence of both measures. However, solely based on the eye tracking results, we would not have been able to conclude that social preference is reduced in the ASD group. Eye tracking conveys where participants are fixating, but it does not provide information about covert processes, possibly leading to reduced sensitivity of the method. Also in this study we observed that a large proportion of time, participants looked in between both stimuli. With frequency-tagging EEG, it is possible to monitor the responses to multiple stimuli that are simultaneously visible. This allows measuring the effects of allocating attention to a spatial location even for stimuli that are outside the (overt) focus of attention. Thanks to this advantage, frequency-tagging is a sensitive method that allowed us to observe a clear pattern in the neural responses. In addition, the time-dependency analyses indicate that the neural signals are highly stable over time. Importantly, the group differences are already present from the earliest seconds of the trials, and persist over the whole duration of the trials, lasting for $30 \mathrm{~s}$. This suggests that further studies could even opt for shorter trial durations. Altogether, these results underline the advantage of frequency-tagging EEG as a sensitive measure of implicit attentional preference, possibly suggesting that its use could contribute to a more coherent literature.

Since the use of multiple simultaneous inputs reduces the time needed for assessments (e.g., example of clinical visual field assessment) (Abdullah, Aldahlawi, Rosli, Boon, \& Maddess, 2012), frequency-tagging EEG can be a very fast and efficient measure. Given the short recording time of the frequency-tagging paradigm and the robustness of the obtained results, the method could be used as a fast marker of social preference, which may ultimately be incorporated in the clinical diagnostic procedure. Furthermore, this has a particular advantage for infant research. Eye tracking requires that the device can reliably capture the eyes of the infant, which is often challenging, resulting in a large amount of missing data.

Pertaining to the choice of the stimulation frequencies, both input frequencies were chosen to be close together to ensure that they would not elicit too large differences in absolute EEG response (Boremanse et al., 2014; Norcia et al., 2015; Regan, 1989). Yet, we did observe a main effect of frequency, indicating that stimuli presented at $6 \mathrm{~Hz}$ generally elicit larger EEG responses as compared to stimuli presented at $7.5 \mathrm{~Hz}$. Importantly, this effect was present regardless of stimulus type and of participant group. These results show that the observed effects were not specific for one of the presentation rate combinations, and underline the importance of counterbalancing the frequencies. The particularly used input frequencies seem valid, but future studies could explore other frequency rates that are even closer together, so that differences in power spectra would be more reduced. Yet, it should be noted that the two frequencies cannot be too close to each other. This is important to ensure that the neighboring 'noise' bins which are used to calculate the signal-to-noise ratio only include noise and no signal of the other presentation frequency.

In a recent study with a partly overlapping subject sample (Vettori et al., 2019), we found no evidence that boys with ASD categorize faces within a stream of objects differently than TD boys. Those results, combined with results from this study, suggest that not merely the difference between faces and objects is altered in boys with ASD, but they tend to prioritize faces less when there is competition between stimuli (which is not the case in the other study).

The use of a well-selected, well-matched and homogeneous participant sample in terms of age, gender, IQ and diagnostic status is certainly an asset. Against this background, one may question whether the findings will generalize to the broader autism population. Further studies will of course be required in order to address this issue. Importantly, however, the advantages of using frequency tagging in combination with eye tracking allowed us to find robust evidence for a reduced social bias in boys with ASD.

\section{Acknowledgements}

This work was supported by grants of the Research Foundation Flanders (FWO; G0C7816N) and Excellence of Science (EOS) grant (G0E8718N; HUMVISCAT) and of the MargueriteMarie Delacroix foundation. Gratitude goes to the participants and their parents for participating in our study. We would also like to thank Dr. Pieter Moors and Dr. Jannes Nys who contributed to this paper through many insightful discussions. 


\section{R E F E R E N C E S}

Abdullah, S. N., Aldahlawi, N., Rosli, Y., Boon, M. Y., \& Maddess, T. (2012). Effect of contrast, stimulus density, and viewing distance on multifocal steady-state visual evoked potentials (MSVs). Investigative ophthalmology \& visual science, 53(9), 5527-5535.

Adrian, E. D., \& Matthews, B. H. C. (1934). The interpretation of potential waves in the cortex. Journal of Physiology, 81, 440-471.

American Psychiatric Association. (2013). Diagnostic and statistical manual of mental disorders (DSM-5). American Psychiatric Pub.

Andersen, S. K., Fuchs, S., \& Müller, M. M. (2011). Effects of feature-selective and spatial attention at different stages of visual processing. Journal of Cognitive Neuroscience, 23, 238-246.

Baayen, R. H., Davidson, D. J., \& Bates, D. M. (2008). Mixed-effects modeling with crossed random effects for subjects and items. Journal of memory and language, 59(4), 390-412.

Bekhtereva, V., Pritschmann, R., Keil, A., \& Müller, M. M. (2018). The neural signature of extracting emotional content from rapid visual streams at multiple presentation rates: A crosslaboratory study. Psychophysiology, 55, e13222.

Bentin, S., Allison, T., Puce, A., Perez, E., \& McCarthy, G. (1996). Electrophysiological studies of face perception in humans. Journal of Cognitive Neuroscience, 8, 551-565.

Boremanse, A., Norcia, A. M., \& Rossion, B. (2014). Dissociation of part-based and integrated neural responses to faces by means of electroencephalographic frequency tagging. The European Journal of Neuroscience, 40, 2987-2997.

Chevallier, C., Kohls, G., Troiani, V., Brodkin, E. S., \& Schultz, R. T. (2012). The social motivation theory of autism. Trends in Cognitive Sciences, 16, 231-239.

Chita-Tegmark, M. (2016). Social attention in ASD: A review and meta-analysis of eye-tracking studies. Research in Developmental Disabilities, 48, 79-93.

Clements, C. C., Zoltowski, A. R., Yankowitz, L. D., Yerys, B. E., Schultz, R. T., \& Herrington, J. D. (2018). Evaluation of the social motivation hypothesis of autism: A systematic review and meta-analysis. JAMA Psychiatry, 75, 797-808.

Constantino, J. N., \& Gruber, C. P. (2012). Social responsiveness scale (SRS). CA: Western Psychological Services Torrance.

Crouzet, S. M., Kirchner, H., \& Thorpe, S. J. (2010). Fast saccades toward faces: Face detection in just 100 ms. Journal of Vision, 10, 16.1-17.

Dawson, G., Carver, L., Meltzoff, A. N., Panagiotides, H., McPartland, J., \& Webb, S. J. (2002). Neural correlates of face and object recognition in young children with autism spectrum disorder, developmental delay, and typical development. Child development, 73(3), 700-717.

Dzhelyova, M., Jacques, C., \& Rossion, B. (2017). At a single glance: Fast periodic visual stimulation uncovers the spatio-temporal dynamics of brief facial expression changes in the human brain. Cerebral Cortex, 27, 4106-4123.

Fischer, J., Smith, H., Martinez-Pedraza, F., Carter, A. S., Kanwisher, N., \& Kaldy, Z. (2016). Unimpaired attentional disengagement in toddlers with autism spectrum disorder. Developmental Science, 19, 1095-1103.

Fletcher-Watson, S., Findlay, J. M., Leekam, S. R., \& Benson, V. (2008). Rapid detection of person information in a naturalistic scene. Perception, 37, 571-583.

Frazier, T. W., Strauss, M., Klingemier, E. W., Zetzer, E. E., Hardan, A. Y., Eng, C., et al. (2017). A meta-analysis of gaze differences to social and nonsocial information between individuals with and without autism. Journal of the American Academy of Child and Adolescent Psychiatry, 56, 546-555.

Freeth, M., Ropar, D., Mitchell, P., Chapman, P., \& Loher, S. (2011). How adolescents with ASD process social information in complex scenes. combining evidence from eye movements and verbal descriptions. Journal of Autism and Developmental Disorders, 41, 364-371.

Goren, C. C., Sarty, M., \& Wu, P. Y. (1975). Visual following and pattern discrimination of face-like stimuli by newborn infants. Pediatrics, 56, 544-549.

Guillon, Q., Hadjikhani, N., Baduel, S., \& Rogé, B. (2014). Visual social attention in autism spectrum disorder: Insights from eye tracking studies. Neuroscience and Biobehavioral Reviews, 42, 279-297.

Halgren, E., Raij, T., Marinkovic, K., Jousmäki, V., \& Hari, R. (2000). Cognitive response profile of the human fusiform face area as determined by MEG. Cerebral Cortex N. Y. N 1991, 10(1), 69-81.

Hershler, O., \& Hochstein, S. (2005). At first sight: A high-level pop out effect for faces. Vision Research, 45, 1707-1724.

Hessels, R. S., Niehorster, D. C., Kemner, C., \& Hooge, I. T. C. (2017). Noise-robust fixation detection in eye movement data: Identification by two-means clustering (I2MC). Behavior Research Methods, 49, 1802-1823.

Jacques, C., Retter, T. L., \& Rossion, B. (2016). A single glance at natural face images generate larger and qualitatively different category-selective spatio-temporal signatures than other ecologically-relevant categories in the human brain. Neuroimage, 137, 21-33.

Johnson, M. H., Dziurawiec, S., Ellis, H., \& Morton, J. (1991). Newborns' preferential tracking of face-like stimuli and its subsequent decline. Cognition, 40, 1-19.

Kang, E., Keifer, C. M., Levy, E. J., Foss-Feig, J. H., McPartland, J. C. \& Lerner, M. D. (2018). Atypicality of the N170 event-related potential in autism spectrum disorder: A meta-analysis. Biological Psychiatry: Cognitive Neuroscience and Neuroimaging, 3, 657-666.

Keil, A., Gruber, T., Müller, M. M., Moratti, S., Stolarova, M., Bradley, M. M., et al. (2003). Early modulation of visual perception by emotional arousal: Evidence from steady-state visual evoked brain potentials. Cognitive, Affective \& Behavioral Neuroscience, 3, 195-206.

Kikuchi, Y., Senju, A., Tojo, Y., Osanai, H., \& Hasegawa, T. (2009). Faces do not capture special attention in children with autism spectrum disorder: A change blindness study. Child Development, 80, 1421-1433.

Kort, W., Schittekatte, M., Dekker, P. H., Verhaeghe, P., Compaan, E. L., Bosmans, M., et al. (2005). WISC-III NL wechsler intelligence scale for children. Derde Editie NL. Handleiding en Verantwoording. Amsterdam Psychologen HTPNIv.

Lenth, R., Singmann, H., Love, J., Buerkner, P., \& Herve, M. (2019). emmeans: Estimated marginal means, aka least-squares means.

Liu-Shuang, J., Norcia, A. M., \& Rossion, B. (2014). An objective index of individual face discrimination in the right occipitotemporal cortex by means of fast periodic oddball stimulation. Neuropsychologia, 52, 57-72.

Morgan, S. T., Hansen, J. C., \& Hillyard, S. A. (1996). Selective attention to stimulus location modulates the steady-state visual evoked potential. Proceedings of the National Academy of Sciences of the United States of America, 93, 4770-4774.

Müller, M. M., Andersen, S., Trujillo, N. J., Valdés-Sosa, P., Malinowski, P., \& Hillyard, S. A. (2006). Feature-selective attention enhances color signals in early visual areas of the human brain. Proceedings of the National Academy of Sciences of the United States of America, 103, 14250-14254.

Niehorster, D. C., Cornelissen, T. H., Holmqvist, K., Hooge, I. T., \& Hessels, R. S. (2018). What to expect from your remote eyetracker when participants are unrestrained. Behavior research methods, 50(1), 213-227.

Nishizato, M., Fujisawa, T. X., Kosaka, H., \& Tomoda, A. (2017). Developmental changes in social attention and oxytocin levels in infants and children. Scientific Reports, 7, 2540. 
Norcia, A. M., Appelbaum, L. G., Ales, J. M., Cottereau, B. R., \& Rossion, B. (2015). The steady-state visual evoked potential in vision research: A review. Journal of Vision, 15, 4-4.

Parish-Morris, J., Chevallier, C., Tonge, N., Letzen, J., Pandey, J., \& Schultz, R. T. (2013). Visual attention to dynamic faces and objects is linked to face processing skills: A combined study of children with autism and controls. Frontiers in Psychology, 4, 185.

Petersen, S. E., \& Posner, M. I. (2012). The attention system of the human brain: 20 Years after. Annual Review of Neuroscience, 35, 73-89.

Pierce, K., Marinero, S., Hazin, R., McKenna, B., Barnes, C. C., \& Malige, A. (2016). Eye tracking reveals abnormal visual preference for geometric images as an early biomarker of an autism spectrum disorder subtype Associated with increased symptom severity. Biological Psychiatry, 79, 657-666.

Posner, M. I., \& Petersen, S. E. (1990). The attention system of the human brain. Annual Review of Neuroscience, 13, 25-42.

Purcell, D. G., \& Stewart, A. L. (1988). The face-detection effect: Configuration enhances detection. Perception Psychophysics, 43, 355-366.

R Core Team. (2018). R: A language and environment for statistical computing. Vienna, Austria: R Foundation for Statistical Computing.

Regan, D. (1966). Some characteristics of average steady-state and transient responses evoked by modulated light. Electroencephalography and Clinical Neurophysiology, 20, 238-248.

Regan, D. (1989). Human brain electrophysiology: Evoked potentials and evoked magnetic fields in science and medicine. Amsterdam, The Netherlands: Elsevier.

Regan, D., \& Heron, J. R. (1969). Clinical investigation of lesions of the visual pathway: a new objective technique. Journal of neurology, neurosurgery, and psychiatry, 32(5), 479.

Retter, T. L., \& Rossion, B. (2016). Uncovering the neural magnitude and spatio-temporal dynamics of natural image categorization in a fast visual stream. Neuropsychologia, 91, $9-28$.

Rinck, M., \& Becker, E. S. (2006). Spider fearful individuals attend to threat, then quickly avoid it: Evidence from eye movements. Journal of Abnormal Psychology, 115, 231-238.

Rosa Salva, O., Farroni, T., Regolin, L., Vallortigara, G., \& Johnson, M. H. (2011). The evolution of social orienting: Evidence from chicks (Gallus gallus) and human newborns. Plos One, 6, e18802.

Rossion, B., \& Jacques, C. (2011). The N170: Understanding the time course of face perception in the human brain. The Oxford Handbook of ERP Components, 115-142.

Rossion, B., Torfs, K., Jacques, C., \& Liu-Shuang, J. (2015). Fast periodic presentation of natural images reveals a robust faceselective electrophysiological response in the human brain. Journal of Vision, 15, 18-18.
Sattler, J. M. (2001). Assessment of children: Cognitive applications (JM Sattler).

Shaffer, R. C., Pedapati, E. V., Shic, F., Gaietto, K., Bowers, K. Wink, L. K., et al. (2017). Brief report: Diminished gaze preference for dynamic social interaction scenes in youth with autism spectrum disorders. Journal of Autism and Developmental Disorders, 47, 506-513.

Shah, P., Gaule, A., Bird, G., \& Cook, R. (2013). Robust orienting to protofacial stimuli in autism. Current Biology CB, 23, R1087-R1088.

Singmann, H., Bolker, B., Westfall, J., \& Aust, F. (2018). afex: Analysis of factorial experiments.

Tanaka, J. W., \& Sung, A. (2016). The "eye avoidance" hypothesis of autism face processing. Journal of autism and developmental disorders, 46(5), 1538-1552.

Thigpen, N. N., Bradley, M. M., \& Keil, A. (2018). Assessing the relationship between pupil diameter and visuocortical activity. Journal of Vision, 18.

Toffanin, P., de Jong, R., Johnson, A., \& Martens, S. (2009). Using frequency tagging to quantify attentional deployment in a visual divided attention task. International Journal of Psychophysiology, 72, 289-298.

Van der Donck, S., Dzhelyova, M., Vettori, S., Thielen, H., Steyaert, J., Rossion, B., et al. (2019). Fast periodic visual stimulation EEG reveals reduced neural sensitivity to fearful faces in children with autism. Journal of Autism and Developmental Disorders, 49(11), 4658-4673.

Vettori, S., Dzhelyova, M., Van der Donck, S., Jacques, C., Steyaert, J., Rossion, B., et al. (2019). Reduced neural sensitivity to rapid individual face discrimination in autism spectrum disorder. NeuroImage Clinical, 21, 101613.

Vettori, S., Jacques, C., Boets, B., \& Rossion, B. (2019). Can the N170 Be used as an electrophysiological biomarker indexing face processing difficulties in autism spectrum disorder? Biological Psychiatry: Cognitive Neuroscience and Neuroimaging, 4(3), 321-323.

Vialatte, F.-B., Maurice, M., Dauwels, J., \& Cichocki, A. (2010). Steady-state visually evoked potentials: Focus on essential paradigms and future perspectives. Progress in Neurobiology, 90, 418-438.

Wechsler, D. (1991). The Wechsler intelligence scale for children (3rd ed.). San Antonio, TX: The Psychological Corporation.

Wieser, M. J., McTeague, L. M., \& Keil, A. (2012). Competition effects OF threatening faces IN social anxiety. Emotion Washington DC, 12, 1050-1060.

Wilson, C. E., Brock, J., \& Palermo, R. (2010). Attention to social stimuli and facial identity recognition skills in autism spectrum disorder. Journal of Intellectual Disability Research JIDR, 54, 1104-1115.

Zeeland, A. A. S.-V., Dapretto, M., Ghahremani, D. G., Poldrack, R. A., \& Bookheimer, S. Y. (2010). Reward processing in autism. Autism Research, 3, 53-67. 